\title{
BMJ Open Is ioflupane I123 injection diagnostically effective in patients with movement disorders and dementia? Pooled analysis of four clinical trials
}

\author{
John T O'Brien, ${ }^{1}$ Wolfgang H Oertel, ${ }^{2}$ Ian G McKeith, ${ }^{3}$ Donald G Grosset, ${ }^{4}$ \\ Zuzana Walker, ${ }^{5,6}$ Klaus Tatsch, ${ }^{7}$ Eduardo Tolosa, ${ }^{8}$ Paul F Sherwin, ${ }^{9}$ \\ Igor D Grachev ${ }^{10}$
}

To cite: O'Brien JT, Oertel WH, McKeith IG, et al. Is ioflupane I123 injection diagnostically effective in patients with movement disorders and dementia? Pooled analysis of four clinical trials. BMJ Open 2014;4:e005122.

doi:10.1136/bmjopen-2014005122

- Prepublication history and additional material is available. To view please visit the journal (http://dx.doi.org/ 10.1136/bmjopen-2014005122)

Received 24 February 2014 Revised 4 June 2014 Accepted 16 June 2014

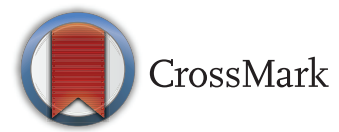

For numbered affiliations see end of article.

\section{Correspondence to}

Professor John T O'Brien; john.obrien@medschl.cam. ac.uk

\section{ABSTRACT}

Objectives: To pool clinical trials of similar design to assess overall sensitivity and specificity of ioflupane I123 injection (DaTSCAN or ioflupane $\left({ }^{123} \mathrm{I}\right)$ ) to detect or exclude a striatal dopaminergic deficit disorder (SDDD), such as parkinsonian syndrome and dementia with Lewy bodies.

Design: Pooled analysis of three phase 3 and one phase 4 clinical trials. These four trials were selected because they were the four studies used for the US new drug application to the Food and Drug Administration (FDA).

Setting: Multicentre, open-label, non-randomised.

Participants: Patients with either a movement disorder or dementia, and healthy volunteers.

Interventions: Ioflupane $\left({ }^{123} \mathrm{I}\right)$ was administered. Outcome measures: Images were assessed by panels of 3-5 blinded experts and/or on-site nuclear medicine physicians, classified as normal or abnormal and compared with clinical diagnosis (reference standard) to determine sensitivity and specificity. Results: Pooling the four studies, 928 participants were enrolled, 849 were dosed and 764 completed their study. Across all studies, when images were assessed by on-site readers, ioflupane $\left({ }^{123} \mathrm{I}\right)$ diagnostic effectiveness had an overall $(95 \% \mathrm{Cl})$ sensitivity of $91.9 \%(88.7 \%$ to $94.5 \%)$ and specificity of $83.6 \%$ $(78.7 \%$ to $87.9 \%)$. When reads were conducted blindly by a panel of independent experts, the overall sensitivity was $88.7 \%(86.8 \%$ to $90.4 \%)$ and specificity was $91.2 \%$ (89.0\% to $93.0 \%$ ).

Conclusions: In this pooled analysis, the visual assessment of ioflupane $\left({ }^{123} \mathrm{l}\right)$ images provided high levels of sensitivity and specificity in detecting the presence/absence of an SDDD. Ioflupane $\left.{ }^{(23} \mathrm{I}\right)$ imaging has the potential to improve diagnostic accuracy in patients with signs and symptoms of a movement disorder and/or dementia.

Trial registration number: NCT00209456.

\section{INTRODUCTION}

Despite the development of consensus clinical diagnostic criteria $^{1-5}$ early and accurate

\section{Strengths and limitations of this study}

- This study provides the largest and most definitive set of clinical evidence to date, summarising experience from three phase 3 and one phase 4 trials with all data pooled for a new statistical analysis, $\mathrm{N}=726$, showing that ioflupane $\left({ }^{123} \mathrm{I}\right)$ singlephoton emission CT imaging has high sensitivity and specificity for detecting the presence or absence of a striatal dopaminergic deficit in patients with movement disorders and dementia (intent to diagnose and per protocol populations). Differences among different patient populations and inter-reader blinded image evaluation results are reported.

- Well-designed, prospective studies with 12-36 months of clinical follow-up after ioflupane $\left({ }^{123} \mathrm{l}\right)$ imaging, in which blinded image evaluation by 3-5 independent nuclear medicine physicians (no access to clinical information) was used for image assessment.

- Studies did not have autopsy confirmation of diagnosis (found to be impractical for up to 36 months of follow-up in the majority of patients in early stage of the disease), though the standard of expert clinical diagnosis, particularly at follow-up after 12 months or later, is an accepted reference standard for biomarker validation studies.

- Only two of the studies (PDT301 and PDT304) used expert clinical panels to establish the clinical diagnosis; the others relied on on-site investigator diagnosis (though made blind to imaging findings, except one clinical utility study PDT408).

diagnosis of common neurodegenerative conditions like Parkinson's disease (PD) and dementia with Lewy bodies (DLB) continues to present challenges. Delays in diagnosis cause unnecessary distress and uncertainty for patients and their families, increase healthcare use through additional appointments and 
investigations and increase the risk that patients will develop preventable disabilities. ${ }^{6}$ Not surprisingly, the longer a patient is observed and the greater the amount of accumulated clinical information, such as response to medications and progression of signs and symptoms, the greater the accuracy of the diagnosis. ${ }^{7}$ Inaccurate diagnoses may result in prescription of inappropriate medications, needlessly exposing patients to potentially harmful side effects, while denying patients treatment of symptoms. ${ }^{6}$ Furthermore, diagnostic discrimination between degenerative and non-degenerative diseases is important because disease course, therapy and prognosis differ considerably among patients. ${ }^{68}$

Differential diagnosis of movement disorders may be confounded by presence of inconsistent parkinsonian features and/or atypical presentation of classic symptoms. Differentiation of Alzheimer's disease (AD) from DLB is also difficult, even after multiple evaluations. Consensus clinical criteria ${ }^{2-5} 9$ without imaging results have good specificity $(80-90 \%)$, but sensitivity is highly variable and can be as low as $30 \%$, with the most common misdiagnosis being AD. ${ }^{9} 10$

The advent of in vivo visualisation of striatal dopamine transporter using the radiopharmaceutical ioflupane $\left({ }^{123} \mathrm{I}\right) \quad$ (iodine-123-fluoropropyl (FP)-carbomethoxy-3 $\beta$ (4-iodophenyltropane) (CIT) or ioflupane I123 injection or $\left[{ }^{123} \mathrm{I}\right]$ ioflupane or $\left[{ }^{123} \mathrm{I}\right]$ FP-CIT or DaTSCAN or DaTscan, GE Healthcare, Amersham, UK) and singlephoton emission CT (SPECT) imaging has enhanced clinicians' ability to differentiate diseases that involve loss of dopaminergic nigrostriatal neurons from those that do not. Throughout this paper we will refer to these disorders as striatal dopaminergic deficit disorders (SDDDs), which is the clinicopathoanatomical term used here as a group term for the clinical reference diagnoses of parkinsonian syndrome (PS) and/or DLB, by virtue of them being recognised as clinical disorders that are known to have striatal dopaminergic deficit. Ioflupane $\left({ }^{123} \mathrm{I}\right)$ is the only approved imaging agent for this purpose; the European Medicines Agency (EMA) approved it under the trade name DaTSCAN (ioflupane $\left.\left({ }^{123} \mathrm{I}\right)\right)$ in $2000,{ }^{11}$ and the US Food and Drug Administration (FDA) approved it under the trade name DaTscan (ioflupane I123 injection) in $2011 .{ }^{12}$ It is currently approved in 33 countries. We searched the literature and found numerous clinical trials that have been performed to establish the technical feasibility, diagnostic effectiveness, sensitivity and specificity of ioflupane $\left({ }^{123} \mathrm{I}\right) \cdot{ }^{13-43}$ However, each trial had limited numbers of participants for whom results were available, ranging from 16 to $326 .{ }^{37}{ }^{15}$ Our search revealed that two meta-analyses have been performed evaluating diagnostic accuracy of SPECT imaging in DLB and in PSs. ${ }^{44} 45$ However, no previous pooled data analysis had been undertaken and the aim of this study was to undertake a pooled analysis using the four clinical studies that were the large, multisite efficacy trials submitted to support the new drug application filing in the USA (three of them for EU) for licensing. They were conducted to the Good Clinical Practice (GCP) standards in predefined populations. Meta-analyses do not allow combination of individual participant's data; only mean values from each study publication are used, rather than maximising information from the raw data. Meta-analyses include all available studies, and may include small, exploratory, non-GCP studies; and may include tracer prototypes (eg, non-approved tracers such as $\beta$-CIT) that are not manufactured to commercial tracer quality, with robust, regulatory-accepted good manufacturing practice processes.

Although two of our studies had been included in each of the meta-analyses (PDT301 baseline ${ }^{14}$ in, ${ }^{44}$ and DP008-003 ${ }^{13}$ in $^{45}$ ), the other two had not. Performing a pooled analysis would provide a large body of evidence on the diagnostic performance of ioflupane $\left({ }^{123} \mathrm{I}\right)$ in participants with movement disorders or dementia.

\section{METHODS}

\section{Participants}

The research question was to determine the pooled diagnostic accuracy (sensitivity and specificity) of the four trials submitted to the US FDA application for ioflupane $\left({ }^{123} \mathrm{I}\right){ }^{13-18}$ All studies tested the effectiveness of ioflupane $\left({ }^{123} \mathrm{I}\right)$ (for the purposes of this report, ioflupane $\left({ }^{123} \mathrm{I}\right)$ will be used throughout the paper) in detecting the loss of dopaminergic nigrostriatal neurons in participants with symptoms and signs of movement disorders and/or dementia. The reference standard was the final clinical diagnosis of a disease that is known to have or not have a striatal dopaminergic deficit (hereafter called reference clinical diagnosis). ${ }^{46}$ This clinical diagnosis was made blind to imaging results in three of the four studies (phase 3 studies DP008-003, PDT301, PDT304 (also elsewhere sometimes known as PDT03004)). In two of the four studies (PDT301 and PDT304), the final clinical diagnosis was made by a panel of experts. Table 1 summarises the attributes of the four studies. Although phase 4 study PDT408 was designed to assess the clinical utility of ioflupane $\left({ }^{123} \mathrm{I}\right)$ image assessments as the primary end point, sensitivity and specificity were secondary end points, and the image results were included in the pooled analysis. The investigators who participated in each of the four studies are listed in online supplementary table S1.

All studies were conducted in accordance with the current revision of the Declaration of Helsinki; the GCP: Consolidated Guideline, approved by the International Conference on Harmonisation and applicable national and local laws. Participants or their guardians gave written informed consent after the aims, methods, anticipated benefits and potential hazards were explained, and prior to starting any study procedures or assessments. The informed consent for each study included a provision for subsequent analyses, of which this pooled analysis is an example. Study PDT301 is 
Table 1 Summary of studies included in pooled analysis

\begin{tabular}{|c|c|c|c|c|}
\hline & \multicolumn{4}{|l|}{ Principal study } \\
\hline & DP008-003 & PDT304 & PDT301 & PDT408 \\
\hline Study design & $\begin{array}{l}\text { Phase } 3 \\
\text { Multicentre, open-label, } \\
\text { non-randomised } \\
\text { Single-dose } \\
\text { Expert clinical diagnosis at baseline } \\
\text { according to published consensus } \\
\text { criteria as the RCD }\end{array}$ & $\begin{array}{l}\text { Phase } 3 \\
\text { Multicentre, open-label, } \\
\text { non-randomised } \\
\text { Repeat-dose (maximum of } \\
\text { 3) } \\
\text { Expert clinical diagnosis at } \\
36 \text { months as the RCD }\end{array}$ & $\begin{array}{l}\text { Phase } 3 \\
\text { Multicentre, open-label, } \\
\text { non-randomised } \\
\text { Single-dose } \\
\text { Expert clinical diagnosis at } 12 \text { months } \\
\text { as the RCD }\end{array}$ & $\begin{array}{l}\text { Phase } 4 \\
\text { Multicentre, open-label, non-randomised } \\
\text { Single-dose } \\
\text { Expert clinical diagnosis at } 24 \text { months as the } \\
\text { RCD }\end{array}$ \\
\hline $\begin{array}{l}\text { Dates study was } \\
\text { conducted }\end{array}$ & August 1997 to February 1998 & - January 1999 to June 2005 & December 2003 to June 2006 & - November 2000 to November 2003 \\
\hline Population & $\begin{array}{l}\text { Healthy volunteers } \\
\text { - Participants with a clinical } \\
\text { diagnosis of: } \\
\text { Parkinson's disease } \\
\text { - Multiple system atrophy } \\
\text { Progressive supranuclear palsy or } \\
\text { - Essential tremor }\end{array}$ & $\begin{array}{l}\text { Healthy volunteers } \\
\text { - Participants with the clinical } \\
\text { features of: } \\
\text { Early Parkinson's disease or } \\
\text { Tremor (mainly essential } \\
\text { tremor) }\end{array}$ & $\begin{array}{l}\text { Participants with dementia (features } \\
\text { of possible DLB or with features of } \\
\text { other dementia }(A D, V a D) \text { ) }\end{array}$ & $\begin{array}{l}\text { Participants with movement disorders (an } \\
\text { uncertain clinical diagnosis as to PS or } \\
\text { non-PS) }\end{array}$ \\
\hline Efficacy objectives & $\begin{array}{l}\text { Primary } \\
\text { Sensitivity and specificity for } \\
\text { detecting or excluding an SDDD } \\
\text { Secondary } \\
\text { - Inter-reader agreement }\end{array}$ & $\begin{array}{l}\text { Primary } \\
\text { Sensitivity and specificity for } \\
\text { detecting or excluding an } \\
\text { SDDD } \\
\text { Secondary } \\
\text { - Inter-reader agreement }\end{array}$ & $\begin{array}{l}\text { Primary } \\
\text { Sensitivity and specificity for detecting } \\
\text { or excluding an SDDD } \\
\text { Secondary } \\
\text { - Inter-reader agreement }\end{array}$ & $\begin{array}{l}\text { Primary* } \\
\text { Impact of ioflupane }\left({ }^{123} \mathrm{I}\right) \text { image assessments } \\
\text { on patient diagnoses, confidence that patient } \\
\text { had PS, and planned management } \\
\text { Secondary } \\
\text { Sensitivity and specificity for detecting or } \\
\text { excluding an SDDD }\end{array}$ \\
\hline $\begin{array}{l}\text { Type of control } \\
\text { Investigational product }\end{array}$ & $\begin{array}{l}\text { No control used } \\
\text { loflupane }\left({ }^{123} \mathrm{l}\right) 111-185 \mathrm{MBq} \\
(3-5 \mathrm{mCi}) \text { iv, } 1 \text { dose }\end{array}$ & $\begin{array}{l}\text { No control used } \\
\text { loflupane }\left({ }^{123} \mathrm{l}\right) 111-185 \mathrm{MBq} \\
(3-5 \mathrm{mCi}) \text { iv, } 3 \text { doses } \\
18 \text { months apart }\end{array}$ & $\begin{array}{l}\text { No control used } \\
\text { loflupane }\left({ }^{123} \mathrm{I}\right) \text { 111-185 MBq }(3-5 \mathrm{mCi}) \\
\text { iv, } 1 \text { dose }\end{array}$ & $\begin{array}{l}\text { No control used } \\
\text { loflupane }\left({ }^{123} \mathrm{l}\right) 111-185 \mathrm{MBq}(3-5 \mathrm{mCi}) \text { iv, } \\
1 \text { dose ( } 73 \text { participants) or } 2 \text { doses } 24 \text { months } \\
\text { apart ( } 14 \text { participants) }\end{array}$ \\
\hline $\begin{array}{l}\text { Number of study } \\
\text { centres }\end{array}$ & 6 & 10 & 40 & 15 \\
\hline $\begin{array}{l}\text { Number of participants } \\
\text { enrolled }\end{array}$ & 250 & 202 & 351 & 125 \\
\hline $\begin{array}{l}\text { Age of ITD population, } \\
\text { range (mean) }\end{array}$ & $40,80(62.7)$ & $33,79(60.4)$ & $54,90(73.9)$ & $25,84(64.2)$ \\
\hline Gender & $62 \%$ male, $38 \%$ female & $56 \%$ male, $44 \%$ female & $57 \%$ male, $43 \%$ female & $58 \%$ male, $42 \%$ female \\
\hline Race & $\begin{array}{l}\text { Caucasian } 98 \% \\
\text { Black } 1 \% \\
\text { Asian }<1 \%\end{array}$ & Caucasian $100 \%$ & Caucasian $100 \%$ & $\begin{array}{l}\text { Caucasian } 99 \% \\
\text { Asian } 1 \%\end{array}$ \\
\hline $\begin{array}{l}\text { Number of participants } \\
\text { evaluable for efficacy }\end{array}$ & 220 & 102 & 288 & 118 \\
\hline $\begin{array}{l}\text { Blinded reads } \\
\text { performed }\end{array}$ & Yes & Yes & Yes & No \\
\hline
\end{tabular}

*Primary objective was to assess clinical utility of ioflupane $\left({ }^{123} \mathrm{I}\right)$ images; however, images were used for pooled efficacy analysis.

AD, Alzheimer's disease; DLB, dementia with Lewy bodies; ITD, intent to diagnose; MBq, megabecquerel; PS, parkinsonian syndrome; RCD, reference clinical diagnosis; SDDD, striatal dopaminergic deficit disorder; $\mathrm{VaD}$, vascular dementia. 
identified in clinicaltrials.gov as NCT00209456. All other trials began enrolling prior to 1 July 2005, the cut-off date for the initiation of the requirement by the International Committee of Medical Journal Editors for trials to be registered, so are not associated with any public database identifiers.

\section{Procedures}

All studies, including each study's inclusion and exclusion criteria, have been published ${ }^{13-18}$; a brief overview of the methods follows. All four studies were open-label, non-randomised, phase 3 or 4 clinical trials to determine the sensitivity (positive per cent agreement (PPA)) and specificity (negative per cent agreement (NPA)) of ioflupane $\left({ }^{123}\right.$ I) SPECT imaging to detect or exclude an SDDD in participants with various movement disorders (PS, including PD, multiple system atrophy (MSA) and progressive supranuclear palsy (PSP); or essential tremor (ET) ) and/or dementia (DLB, AD or vascular dementia $(\mathrm{VaD}))$, and healthy volunteers. Participants received either a single or repeat (up to three doses total) dose of 111-185 MBq of ioflupane $\left({ }^{123} \mathrm{I}\right)$. SPECT imaging was performed between 3 and $6 \mathrm{~h}$ after injection. Ioflupane $\left({ }^{123} \mathrm{I}\right)$ images were read on-site (institutional reads), as well as by three or five independent blinded readers (blinded image evaluation (BIE)) in three of the studies, and classified as normal (SDDD absent) or abnormal (SDDD present). Abnormal images were further classified as type 1, 2 or $3 .^{12}$ Expert clinical diagnosis using a blinded panel of three neurologists or dementia specialists established whether the participant had an SDDD (PD, PS, PSP, MSA or DLB) or a non-SDDD (ET, AD or VaD and healthy volunteers). Expert clinical diagnosis was established at various time points across the four studies: DP008-003 at baseline, PDT301 at baseline and month 12, PDT408 at baseline and month 24 and PDT304 at baseline and months 18 and 36. In PDT408, the final diagnosis was made with access to the ioflupane $\left({ }^{123} \mathrm{I}\right)$ SPECT images.

Each ioflupane $\left({ }^{123} \mathrm{I}\right)$ image result was compared with the corresponding reference clinical diagnosis, and classified as a true-positive (TP), true-negative (TN), false-positive (FP) or false-negative (FN) scan to allow calculation of sensitivity and specificity. Sensitivity was calculated as $n T P /(n T P+n F N)$, ( $n=$ number of participants). Specificity was calculated as $n T N /(n T N+n F P)$.

Additional efficacy end points included inter-reader agreement between BIE readers, as well as BIE readers versus on-site institutional readers (DP008-003, PDT304 and PDT301).

\section{Statistical analysis}

All statistical analyses were performed using Statistical Analysis Software (SAS Institute Inc, Cary, North Carolina, USA). Demographic data were collected and are presented using descriptive statistics. Populations analysed included enrolled (all participants who were enrolled in any one of the four studies), dosed (all enrolled participants who received ioflupane $\left.\left({ }^{123} \mathrm{I}\right)\right)$, intent to diagnose (ITD; all dosed participants who underwent SPECT imaging and underwent the reference clinical diagnosis assessment for the relevant analysis) and per protocol (PP; all participants in the ITD population with no major protocol violations). Sensitivity and specificity were calculated for the ITD and PP populations, and are reported with 95\% CIs. For the purpose of this report, we will be using sensitivity and specificity (equivalent to PPA and NPA). Pairwise inter-reader and BIE versus on-site reader agreement were analysed using Cohen's $\kappa$ statistic. Inter-reader agreement across all BIE readers was analysed using Fleiss' $\kappa$ statistic.

\section{RESULTS}

\section{Participant disposition and characteristics}

Participant disposition for each study and for the pooled analysis is shown in figure 1 . Of the 928 participants enrolled, 849 (91\%) were dosed, and 764 (82\%) completed their study. The most common reasons for not completing a study included participant request/withdrew consent (85 participants, 9\%), lost to follow-up (34 participants, $4 \%$ ) and protocol violation (14 participants, $2 \%)$. Eleven participants (1\%) did not complete due to safety concerns, including adverse events.

Medical history data were not collected consistently across studies and could not be pooled for this analysis.

By-study and pooled participant baseline demographics are shown in table 2 (ITD population; PP population in online supplementary table S3). No meaningful differences were noted in baseline demographics between the ITD and PP populations. Age was similar in three of the four studies, with participants in PDT301 being olderunsurprisingly because this study only included people with dementia. In all studies, there were more men than women, with a similar ratio across studies. The majority was Caucasian, with African-Americans and/or Asians representing $1 \%$ or less in any single study. Clinical diagnoses represented in each study are tabulated in table 2 (ITD population) and online supplementary table S4 (PP population), and are presented graphically in figure 2A (ITD population) and figure 2B (PP population). Overall, 393 (54\%) of participants in the ITD population were classified as having SDDD (SDDD present), while $249(34 \%)$ were classified with conditions that did not have an SDDD (SDDD absent).

\section{Sensitivity (PPA) and specificity (NPA)}

Sensitivity and specificity for ioflupane $\left({ }^{123} \mathrm{I}\right)$ to detect SDDD (abnormal scan) or non-SDDD (normal scan) using the mean of BIE reads is displayed in figure 3. Online supplementary tables S4 and S5 (ITD and PP populations, respectively) show the means and 95\% CI for the individual reads for PSs, DLB and total. Figure 3A shows high sensitivity and specificity in the ITD population for both movement disorders (PS) and the total pooled analysis, with a slightly lower sensitivity value 


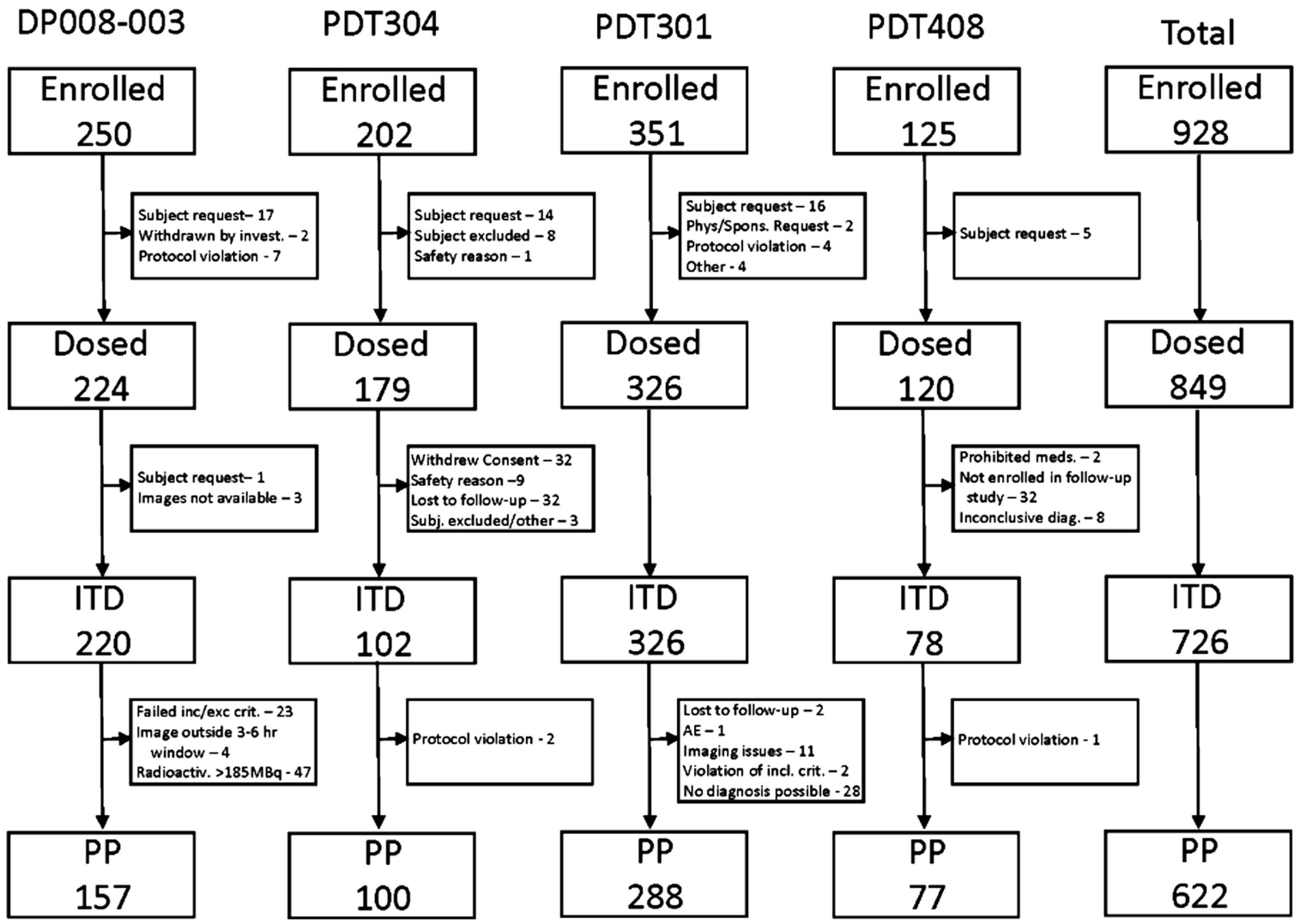

Note: Subjects may have more than one reason for discontinuing.

Figure 1 Participant disposition. AE, adverse event; ITD, intent to diagnose; PP, per protocol.

$(78.5 \%)$ when assessing participants with dementia. Sensitivity and specificity did not change substantially when reference clinical diagnoses were made for DLB at month 12. Sensitivity decreased when reference clinical diagnoses were made for PS at months 18 and 36 (78.9\% and $76.6 \%$ ), but specificity values increased slightly, exceeding $95 \%$ at each time point. Overall, the sensitivity of BIE reads of ioflupane $\left({ }^{123} \mathrm{I}\right)$ SPECT images in the ITD population for PS and dementia at all diagnosis time points ranged from $76.6 \%$ to $91.1 \%$, and specificity ranged from $90.1 \%$ to $96.7 \%$; $\mathrm{PP}$ population results (figure 3C, D) were very similar. Figure 4A-D displays the same analyses using the on-site read results. Overall, sensitivity in the ITD population (figure 4A, B) ranged from $81.4 \%$ to $89.9 \%$, and tended to be higher for on-site reads compared with the BIE reads. Specificity ranged from $81.6 \%$ to $90.3 \%$, and tended to be lower compared with BIE reads. No meaningful differences were noted in the values when analysing the PP population (figure 4C, D). Tables 3 and 4 (ITD and PP populations, respectively) summarise the sensitivity and specificity by expert clinical diagnosis for on-site, institutional reads.

\section{Inter-reader agreement}

Three of the studies had BIE readers, and study PDT304 had three sets of images to be read. Overall, the agreement between the BIE reader pairs was good, and ranged from 0.81 (95\% CI 0.73 to 0.90$)$ to 1.00 (1.00 to $1.00)$. The Fleiss' $\kappa$ for all BIE readers in a study ranged from 0.88 (0.84 to 0.92$)$ to $0.99 \quad(0.87$ to 1.10$)$. Agreement between the BIE readers and the on-site read was similar for two of the studies, and ranged from 0.82 (0.73 to 0.90$)$ to 0.94 (0.87 to 1.01$)$; for study PDT301, the agreement for this comparison was not as good, with $\kappa$ ranging from 0.60 (0.51 to 0.69$)$ to 0.68 (0.60 to 0.76$)$. Inter-reader agreement for the PP population was comparable to that determined for the ITD population (data not shown).

\section{DISCUSSION}

The current pooled analysis provides the largest data set of clinical evidence ( $\mathrm{N}=726$ in the ITD population) to date showing that ioflupane $\left({ }^{123} \mathrm{I}\right)$ SPECT imaging has high sensitivity and specificity for detecting the presence or absence of a striatal dopaminergic deficit in ITD and PP population of patients with movement disorders and/or dementia. Another strength of this study is that we pooled well-designed, prospective studies with 1236 months of clinical follow-up after ioflupane $\left({ }^{123} \mathrm{I}\right)$ imaging in which BIE by 3-5 independent nuclear medicine physicians (no access to clinical information) was used for image assessment. Overall, sensitivity for 
detecting the presence or absence of an SDDD ranged from $75 \%$ to $96.5 \%$, and specificity ranged from $83 \%$ to $100 \%$. Inter-reader agreement was high, with $\kappa$ for blinded reader pairs ranging from 0.81 to 1 , indicating that diagnostic accuracy is not dependent on individual expert performance.

When BIE reads were compared with on-site reads, specificity was higher for the BIE reads, whereas sensitivity was higher for the on-site reads. BIE versus on-site reader agreement was lower in the PDT301 study. This study focused on participants with dementia, whereas the other studies focused primarily on participants with movement disorders. Clinical diagnosis of DLB tends to be less accurate than PS. ${ }^{10} 131647$ On-site readers had access to patient clinical information, whereas BIE readers did not. This likely contributed to the observed increase in sensitivity and decrease in specificity when images were read by the on-site readers compared with BIE readers, resulting in lower agreement between the two reader groups in this study.

A limitation of this study is that the four studies in the pooled analysis used expert clinical diagnosis as a reference standard for the presence or absence of an SDDD. Two of the studies (PDT301 and PDT304) used expert panels to establish the clinical diagnosis. In DP008-003, enrolled participants had established diagnoses, so an expert panel was not considered necessary. In PDT408, the final diagnosis was made with access to the ioflupane $\left({ }^{123} \mathrm{I}\right)$ SPECT images, which was required to assess the test clinical utility. The truth standard for diagnosing movement disorders and dementia is neuropathological confirmation of brain tissue at autopsy. However, with a slowly progressive, mostly benign course of these disorders, these patients are unlikely to die during the course of relatively short clinical trial duration and be participants for autopsy assessment. Previous postmortem studies demonstrated a good correlation between ioflupane $\left({ }^{123}\right.$ I) SPECT imaging with neuropathological findings. ${ }^{19}{ }^{46}$ In a study by Walker et $a l,{ }^{19}$ when validation was by autopsy diagnosis, sensitivity and specificity of initial clinical diagnoses in DLB were $75 \%$ and $42 \%$, respectively, whereas sensitivity and specificity of ioflupane $\left({ }^{123} \mathrm{I}\right)$ imaging were higher, with values of $88 \%$ and $83 \%$, respectively $88 \%$ and $100 \%$ for semiquantitative analysis of scans). Therefore, the use of clinical diagnosis as the non-perfect reference standard rather than neuropathological confirmation at autopsy may have contributed to the sensitivity and specificity values obtained in this pooled analysis. Another limitation of the study is that study PDT408 was not designed specifically to assess the sensitivity and specificity of ioflupane $\left({ }^{123}\right.$ I) SPECT imaging for detecting or excluding an

Table 2 Demographic characteristics and clinical diagnosis (per reference clinical diagnosis) by study —ITD population $(\mathrm{N}=726)$

\begin{tabular}{llllll}
\hline & Study & & & \\
& DP008-003 (N=220) & PDT304 (N=102) & PDT301 (N=326) & PDT408 (N=78) & Total (N=726) \\
\hline Age (year) & & & & & \\
Mean (SD) & $62.7(8.87)$ & $60.4(10.91)$ & $73.9(7.17)$ & $64.2(11.99)$ & $67.6(10.60)$ \\
Minimum, Maximum & 40,80 & 33,79 & 54,90 & 25,84 & 25,90 \\
Median & 63.5 & 61.0 & 75.0 & 67.0 & 69.0 \\
Gender & & & & \\
Male & $136(62 \%)$ & $57(56 \%)$ & $187(57 \%)$ & $41(53 \%)$ & $421(58 \%)$ \\
Female & $84(38 \%)$ & $45(44 \%)$ & $139(43 \%)$ & $37(47 \%)$ & $305(42 \%)$ \\
Race & & & & $77(99 \%)$ & $721(99 \%)$ \\
Caucasian & $216(98 \%)$ & $102(100 \%)$ & $326(100 \%)$ & $0(0 \%)$ & $3(<1 \%)$ \\
Black & $3(1 \%)$ & $0(0 \%)$ & $0(0 \%)$ & $1(1 \%)$ & $2(<1 \%)$ \\
Asian & $1(<1 \%)$ & $0(0 \%)$ & $0(0 \%)$ & $0(0 \%)$ & $0(0 \%)$ \\
Other & $0(0 \%)$ & $0(0 \%)$ & $0(0 \%)$ & $48(62 \%)$ & $277(38 \%)$ \\
PS (SDDD) & $158(72 \%)$ & $71(70 \%)$ & $0(0 \%)$ & $48(62 \%)$ & $211(29 \%)$ \\
Possible PS & $158(72 \%)$ & $5(5 \%)$ & $0(0 \%)$ & $0(0 \%)$ & $66(9 \%)$ \\
Probable PS & $0(0 \%)$ & $66(65 \%)$ & $0(0 \%)$ & $0(0 \%)$ & $116(16 \%)$ \\
DLB (SDDD) & $0(0 \%)$ & $0(0 \%)$ & $116(36 \%)$ & $0(0 \%)$ & $27(4 \%)$ \\
Possible DLB & $0(0 \%)$ & $0(0 \%)$ & $27(8 \%)$ & $0(0 \%)$ & $89(12 \%)$ \\
Probable DLB & $0(0 \%)$ & $0(0 \%)$ & $89(27 \%)$ & $30(38 \%)$ & $249(34 \%)$ \\
Non-PS/non-DLB (no SDDD) & $62(28 \%)$ & $31(30 \%)$ & $126(39 \%)$ & $23(29 \%)$ & $64(9 \%)$ \\
ET & $27(12 \%)$ & $14(14 \%)$ & $0(0 \%)$ & $0(0 \%)$ & $125(17 \%)$ \\
AD & $0(0 \%)$ & $0(0 \%)$ & $125(38 \%)$ & $7(9 \%)$ & $60(8 \%)$ \\
Other & $35(16 \%)$ & $17(17 \%)$ & $1(<1 \%)$ & $48(62 \%)$ & $393(54 \%)$ \\
SDDD present & $158(72 \%)$ & $71(70 \%)$ & $116(36 \%)$ & $30(38 \%)$ & $249(34 \%)$ \\
SDDD absent & $62(28 \%)$ & $31(30 \%)$ & $126(39 \%)$ & &
\end{tabular}

*Includes possible and probable PS and possible and probable DLB diagnoses.

AD, Alzheimer's disease; DLB, dementia with Lewy bodies; ET, essential tremor; ITD, intent to diagnose; N, number of participants in the study; PS, parkinsonian syndrome; SDDD, striatal dopaminergic deficit disorder. 
Figure 2 Summary of clinical diagnosis (per reference clinical standard) by study. (A) ITD population. (B) PP population. AD, Alzheimer's disease; DLB, dementia with Lewy bodies; ET, essential tremor; ITD, intent to diagnose; PP, per protocol; PS, parkinsonian syndrome; SDD, striatal dopaminergic deficit.

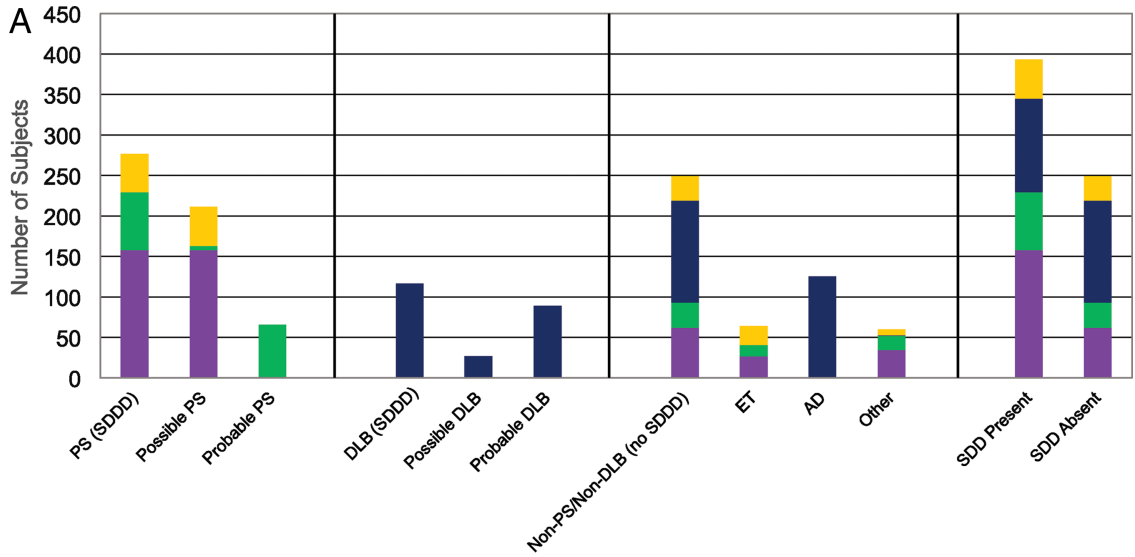

B

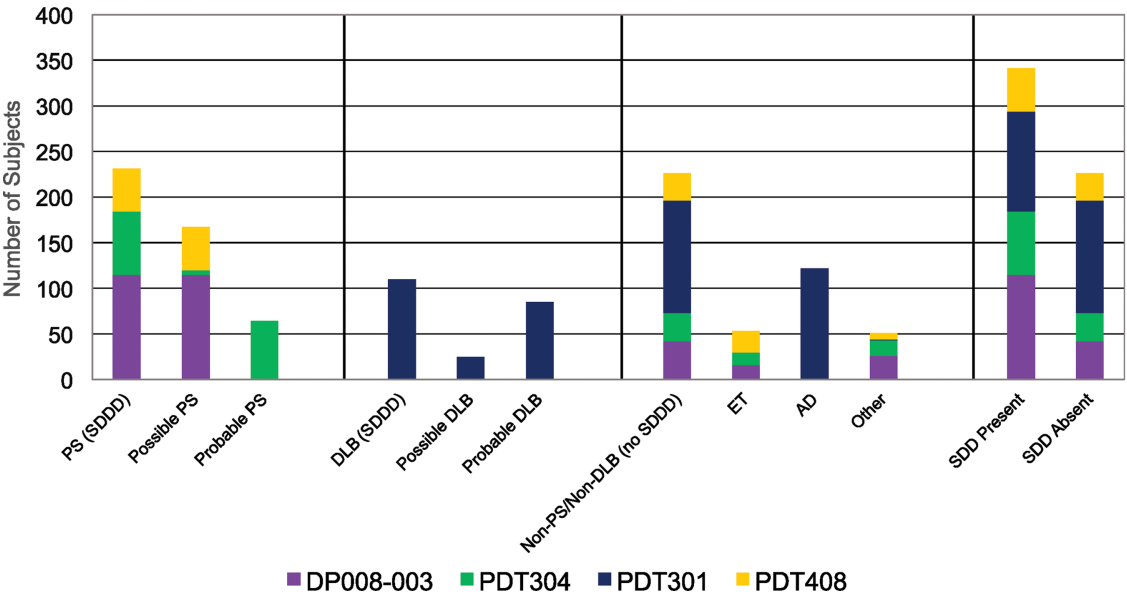

SDDD. However, they were secondary end points, and expert clinical diagnosis and ioflupane $\left({ }^{123} \mathrm{I}\right)$ images were available on these participants, so it was deemed appropriate to include this study in the pooled analysis. Of note, the sensitivity and specificity values for this study fell within the range for the other three studies in which clinical diagnoses were made blinded to ioflupane $\left({ }^{123} \mathrm{I}\right)$ images, and exclusion of this study would not have altered the main findings reported here.

Substantial clinical need has been established for an adjunct to existing diagnostic tools for differentiating PD from ET, and DLB from AD. Examiner expertise affects
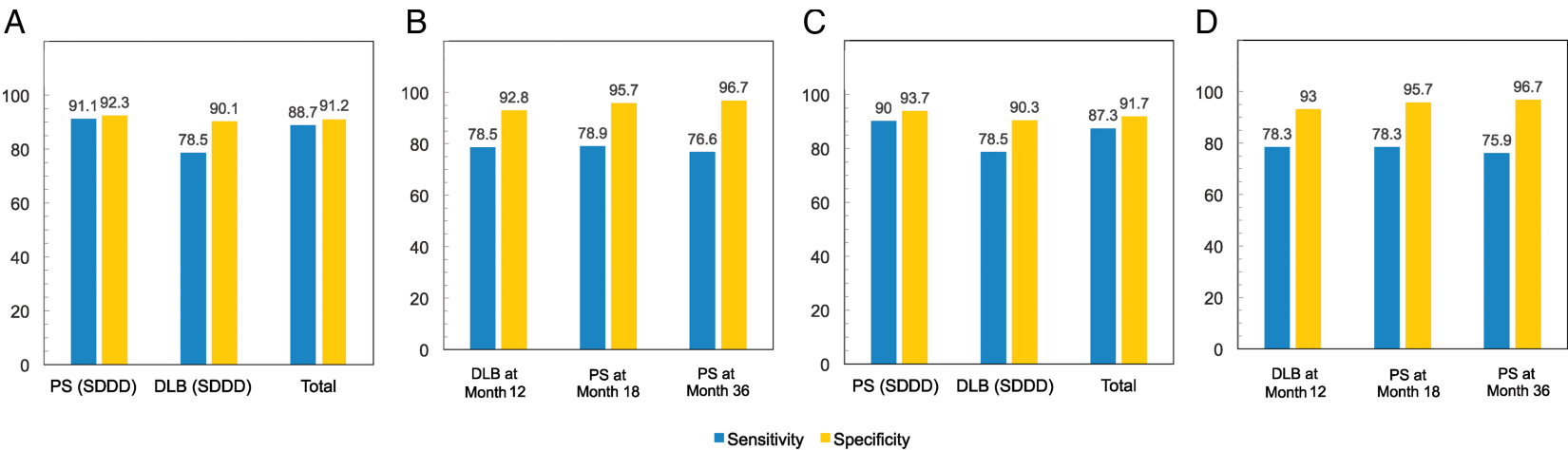

Figure 3 Summary of sensitivity (PPA) and specificity (NPA) by expert clinical diagnosis—mean of blind reads. (A) ITD population-summary results calculated across all studies and readers at baseline. DLB is calculated based on probable DLB versus non-DLB. Total is calculated based on SDDD present versus SDDD absent. (B) ITD population-DLB at month 12 calculated for all readers in study PDT301. PS at month 18 and 36 calculated for all readers in study PDT304. (C) PP population - summary results calculated across all studies and readers at baseline. DLB is calculated based on probable DLB versus non-DLB. Total is calculated based on SDDD present versus SDDD absent. (D) PP population-DLB at month 12 calculated for all readers in study PDT301. PS at month 18 and 36 calculated for all readers in study PDT304. DLB, dementia with Lewy bodies; ITD, intent to diagnose; NPA, negative per cent agreement; PP, per protocol; PPA, positive per cent agreement; PS, parkinsonian syndrome; SDDD, striatal dopaminergic deficit disorder. 

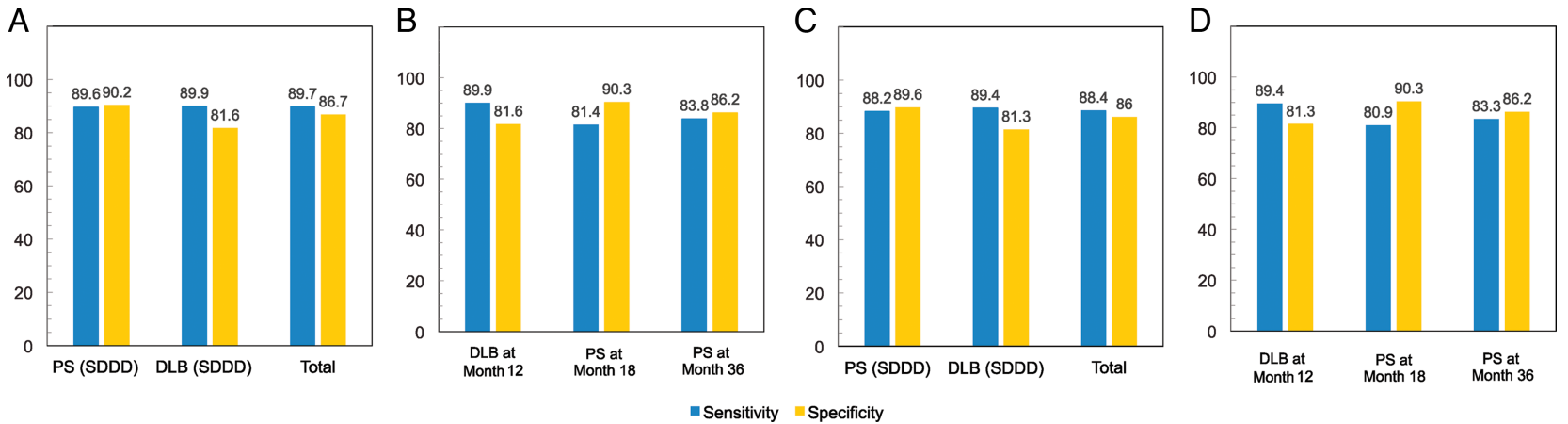

Figure 4 Summary of sensitivity (PPA) and specificity (NPA) by expert clinical diagnosis—on-site institutional reads. (A) ITD population—summary results calculated across all studies and time points. For PDT301, month 12 reference clinical diagnosis was used in this analysis. DLB is calculated based on probable DLB versus non-DLB. Total is calculated based on SDDD present versus SDDD absent. (B) ITD population-DLB at month 12 calculated for on-site readers in study PDT301. PS at month 18 and 36 calculated for on-site readers in study PDT304. (C) PP population-summary results calculated across all studies and time points. For PDT301, month 12 reference clinical diagnosis was used in this analysis. DLB is calculated based on probable DLB versus non-DLB. Total is calculated based on SDDD present versus SDDD absent. (D) PP population-DLB at month 12 calculated for on-site readers in study PDT301. PS at month 18 and 36 calculated for on-site readers in study PDT304. DLB, dementia with Lewy bodies; ITD, intent to diagnose; NPA, negative per cent agreement; PP, per protocol; PPA, positive per cent agreement; PS, parkinsonian syndrome; SDDD, striatal dopaminergic deficit disorder.

diagnostic accuracy, with subspecialists having the highest accuracy, followed by general neurologists; primary care physicians tend to have the lowest. ${ }^{48}$ In a general practice setting $(\mathrm{N}=202), 15 \%$ of patients who had been diagnosed with parkinsonism had tremor with onset after the age of 50 , or who had ever received parkinsonism drugs had their diagnosis unequivocally rejected when strict clinical diagnostic criteria were applied and they completed a detailed neurological interview. ${ }^{23}$ On the other hand, 13 patients $(19 \%)$ not previously diagnosed with PD received this diagnosis following use of strict clinical diagnostic criteria. ${ }^{49}$ In another general practice setting in Scotland $(\mathrm{N}=610)$, $5 \%$ of patients taking antiparkinson therapy for a diagnosis of PD had their medication successfully withdrawn following evaluation by two movement disorder specialists; ioflupane $\left({ }^{123} \mathrm{I}\right)$ scanning was performed if there was uncertainty. ${ }^{50}$ General neurologists changed the diagnosis in $75 \%$ and movement disorder specialists in $47 \%$ of clinically uncertain PS cases after ioflupane $\left({ }^{123} \mathrm{I}\right)$ imaging results became available. ${ }^{6}{ }^{51}$ These studies highlight the frequency of PD or PS misdiagnosis, and illustrate how using ioflupane $\left({ }^{123} \mathrm{I}\right)$ scanning can result in corrections to treatment. Early diagnosis is confounded by the fact that these diseases are progressive, and it may take time for the signs and symptoms to worsen until they clearly point to one disease. ${ }^{7}$ The choice of consensus criteria also affects the sensitivity and specificity of the clinical diagnosis. ${ }^{52}{ }^{53}$ All these factors contribute to clinical diagnosis failing to align with autopsy findings up to $25 \%$ of the time.$^{52}$ Ioflupane $\left({ }^{123}\right.$ I) SPECT imaging does not diagnose disease; rather, it is used to determine the presence or absence of a striatal dopaminergic deficit. The performance of ioflupane $\left({ }^{123} \mathrm{I}\right)$ reported here may have been lower than expected, particularly in patients with DLB, because we were comparing it to clinical diagnosis based on consensus criteria, known to be imprecise.

Regulatory approval of ioflupane $\left({ }^{123} \mathrm{I}\right)$ in Europe and the USA has facilitated meeting the clinical need to improve the accuracy of clinical diagnosis. Adoption and utilisation of this new technology is expanding, and several professional societies and organisations are supporting ioflupane $\left({ }^{123} \mathrm{I}\right)$ imaging as a useful and validated diagnostic tool. These include mention in the 2013 EFNS/MDS-ES/ENS guideline (Category A), ${ }^{54}$ the Society of Nuclear Medicine, ${ }^{55}$ the UK's National Institute for Health and Care Excellence (NICE) 2006 guidance, ${ }^{56}$ the Scottish Intercollegiate Guidelines Network (SIGN) ${ }^{57}$ and the EFNS-ENS guidelines. ${ }^{4}$ The Parkinson Progression Marker Initiative (PPMI) is adding ioflupane $\left({ }^{123} \mathrm{I}\right)$ imaging to be included in study inclusion criteria, as well as during a 5-year study of PD biomarker progression. ${ }^{58}$

Research is needed to more fully elucidate future applications of ioflupane $\left({ }^{123} \mathrm{I}\right)$ SPECT imaging. While not currently licensed for this application, discussions have recently focused on the possibility of whether quantitative analysis of ioflupane $\left({ }^{123} \mathrm{I}\right)$ binding might further increase the sensitivity and specificity of SDDD detection and enable differentiation of other PS, such as PSP, MSA or vascular parkinsonism from PD. ${ }^{20} 5960$ Additional studies that compare ioflupane $\left({ }^{123} \mathrm{I}\right)$ imaging results with postmortem neuropathology rather than expert clinical diagnosis may document better the accuracy of estimates of sensitivity and specificity. Our use of expert clinical diagnosis as the standard of truth, while validated, was not as perfect as autopsy. In addition, not all patients with DLB have nigrostriatal degeneration, and a small percentage of these patients may have primarily cortical degeneration. ${ }^{61}$ Finally, ioflupane $\left({ }^{123} \mathrm{I}\right)$ imaging may be helpful in identifying dopaminergic nigrostriatal 
Table 3 Summary of sensitivity (PPA) and specificity (NPA) by expert clinical diagnosis—on-site institutional reads-ITD population (N=726)

\begin{tabular}{|c|c|c|c|c|c|c|}
\hline \multirow[b]{3}{*}{ Response } & \multicolumn{6}{|c|}{ Expert clinical diagnosis } \\
\hline & \multicolumn{2}{|c|}{ PS; SDDD } & \multicolumn{2}{|l|}{ DLB; SDDD } & \multicolumn{2}{|l|}{ Total } \\
\hline & $\begin{array}{l}\text { Sensitivity } \\
(\%, 95 \% \mathrm{Cl})\end{array}$ & $\begin{array}{l}\text { Specificity } \\
(\%, 95 \% \mathrm{Cl})\end{array}$ & $\begin{array}{l}\text { Sensitivity } \\
(\%, 95 \% \text { Cl) }\end{array}$ & $\begin{array}{l}\text { Specificity } \\
(\%, 95 \% \mathrm{Cl})\end{array}$ & $\begin{array}{l}\text { Sensitivity } \\
(\%, 95 \% \mathrm{Cl})\end{array}$ & $\begin{array}{l}\text { Specificity } \\
(\%, 95 \% \text { Cl) }\end{array}$ \\
\hline Pooled studies* & 93.1 (89.5 to 95.8$)$ & 91.1 (84.6 to 95.5$)$ & $88.3(80.0$ to 94.0$)$ & 77.4 (69.7 to 83.9$)$ & 91.9 (88.7 to 94.5$)$ & 83.6 (78.7 to 87.9$)$ \\
\hline Study PDT301-month 12 & & & 89.9 (81.7 to 95.3$)$ & 81.6 (73.7 to 88.0$)$ & & \\
\hline Study PDT304-month 18 & 81.4 (70.3 to 89.7$)$ & 90.3 (74.2 to 98.0$)$ & & & & \\
\hline Study PDT304-month 36 & 83.8 (72.9 to 91.6$)$ & 86.2 (68.3 to 96.1$)$ & & & & \\
\hline Mean results† & 89.6 (86.3 to 92.4$)$ & 90.2 (84.9 to 94.1$)$ & 89.9 (81.7 to 95.3$)$ & 81.6 (73.7 to 88.0$)$ & 89.7 (86.7 to 92.2) & 86.7 (82.4 to 90.3 ) \\
\hline
\end{tabular}

Sensitivity/specificity for DLB is calculated based on probable DLB versus non-DLB.

Sensitivity/specificity for total is calculated based on SDDD versus non-SDDD.

${ }^{*}$ Pooled studies include on-site ioflupane $\left({ }^{123}\right)$ reads for DP008-003, PDT304 (at baseline), PDT301 (baseline reference clinical diagnosis) and PDT408.

†Summary results calculated across all studies and time points. For PDT301, the month 12 reference clinical diagnosis was used.

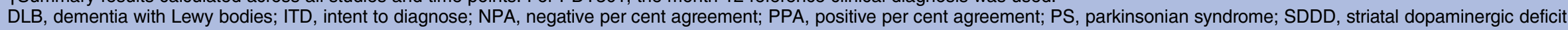
disorder.

Table 4 Summary of sensitivity (PPA) and specificity (NPA) by expert clinical diagnosis—on-site institutional reads-PP population (N=622)

\begin{tabular}{|c|c|c|c|c|c|c|}
\hline \multirow[b]{3}{*}{ Response } & \multicolumn{6}{|c|}{ Expert clinical diagnosis } \\
\hline & \multicolumn{2}{|c|}{ PS; SDDD } & \multicolumn{2}{|l|}{ DLB; SDDD } & \multicolumn{2}{|l|}{ Total } \\
\hline & $\begin{array}{l}\text { Sensitivity } \\
(\%, 95 \% \mathrm{Cl})\end{array}$ & $\begin{array}{l}\text { Specificity } \\
(\%, 95 \% \mathrm{CI})\end{array}$ & $\begin{array}{l}\text { Sensitivity } \\
(\%, 95 \% \mathrm{Cl})\end{array}$ & $\begin{array}{l}\text { Specificity } \\
(\%, 95 \% \mathrm{CI})\end{array}$ & $\begin{array}{l}\text { Sensitivity } \\
(\%, 95 \% \mathrm{CI})\end{array}$ & $\begin{array}{l}\text { Specificity } \\
(\%, 95 \% \mathrm{Cl})\end{array}$ \\
\hline Pooled studies* & 91.8 (87.5 to 95.0$)$ & 90.3 (82.9 to 95.2$)$ & 87.5 (78.7 to 93.6$)$ & 77.1 (69.3 to 83.7 ) & 90.6 (86.8 to 93.6$)$ & 82.6 (77.3 to 87.1$)$ \\
\hline Study PDT301—month 12 & & & 89.4 (80.8 to 95.0$)$ & 81.3 (73.3 to 87.8$)$ & & \\
\hline Study PDT304-month 18 & 80.9 (69.5 to 89.4$)$ & 90.3 (74.2 to 98.0$)$ & & & & \\
\hline Study PDT304-month 36 & 83.3 (72.1 to 91.4$)$ & 86.2 (68.3 to 96.1$)$ & & & & \\
\hline Mean results $\dagger$ & 88.2 (84.5 to 91.3 ) & 89.6 (83.8 to 93.8 ) & 89.4 (80.8 to 95.0$)$ & 81.3 (73.3 to 87.8$)$ & 88.4 (85.1 to 91.2$)$ & $86.0(81.4$ to 89.8$)$ \\
\hline
\end{tabular}

Sensitivity/specificity for DLB is calculated based on probable DLB versus non-DLB.

Sensitivity/specificity for total is calculated based on SDDD versus non-SDDD.

${ }^{*}$ Pooled studies include on-site [ ${ }^{123}$ |] FP-CIT reads for DP008-003, PDT304 (at baseline), PDT301 (baseline reference clinical diagnosis) and PDT408.

†Summary results calculated across all studies and time points. For PDT301, the month 12 reference clinical diagnosis was used.

DLB, dementia with Lewy bodies; FP-CIT, fluoropropyl-carbomethoxy-3ß-(4-iodophenyltropane); NPA, negative per cent agreement; PP, per protocol; PPA, positive per cent agreement; PS,

parkinsonian syndrome; SDDD, striatal dopaminergic deficit disorder. 
degeneration in the prodromal stages, such as rapid-eye-movement sleep behaviour disorder of $\alpha$-synucleinopathies (PD, MSA, DLB) and tauopathies (PSP, corticobasal degeneration). ${ }^{62} 63$

\section{Author affiliations}

${ }^{1}$ Department of Psychiatry, University of Cambridge School of Clinical Medicine, and Cambridgeshire and Peterborough Foundation NHS Trust, Cambridge, UK

${ }^{2}$ Department of Neurology, Philipps-University of Marburg, Marburg, Germany ${ }^{3}$ Campus for Ageing and Vitality, Newcastle University, Newcastle upon Tyne, UK

${ }^{4}$ Department of Neurology, Institute of Neurological Sciences, Southern General Hospital, and University of Glasgow, Glasgow, Scotland, UK ${ }^{5}$ Mental Health Sciences Unit, University College London, London, UK ${ }^{6}$ North Essex Partnership University NHS Foundation Trust, Essex, UK ${ }^{7}$ Department of Nuclear Medicine, Municipal Hospital Karlsruhe, Inc., Karlsruhe, Germany

${ }^{8}$ Neurology Service, Hospital Clínic, University of Barcelona, Institut d'investigacions Biomédiques August Pi i Sunyer (IDIBAPS), Centro de Investigación Biomédica en Red de Enfermedades Neurodegenerativas (CIBERNED), Barcelona, Catalonia, Spain

${ }^{9}$ Clinical Development, Life Sciences, GE Healthcare, Princeton, New Jersey, USA

${ }^{10} \mathrm{Global}$ Head of Neurology/DaTscan, Medical Affairs, Life Sciences, GE Healthcare, Princeton, New Jersey, USA

Acknowledgements The authors would like to acknowledge the writing assistance provided by Stacy Simpson Logan, CMPP of Winfield Consulting, and funded by GE Healthcare. They thank the patients and their families, who selflessly agreed to study participation. JT O'B was supported by the NIHR Dementia Biomedical Research Unit and Centre awarded to the University of Cambridge and Cambridgeshire and Peterborough NHS Foundation Trust and Cambridge University Hospitals NHS Foundation Trust.

Contributors JTO'B was a principal investigator responsible for design, conduct and aspects of data collection and supervision of the 301 study; he was involved in design and critical analysis of data forming this manuscript. WHO contributed to the study designs, data collection, data analysis and data interpretation. IGM and ZW contributed to data collection. DGG made substantial contribution to the acquisition, analysis and interpretation of the data. KT was involved in the analysis and reporting of study results, which are presented in this manuscript (investigator and reader in part of the studies), as well as contributing to the interpretation of the data in this pooled analysis ET contributed to the study design, data analysis and data interpretation. PFS was involved in reporting of studies that resulted in data reported in this manuscript, as well as contributing to the analysis and interpretation of this pooled analysis. IDG provided funding and administrative support; managed statistical analysis and medical writing; conducted literature search; interpreted the data and drafted the first draft and efficacy sections of the manuscript. All authors reviewed and edited the manuscript, and approved the final version. All authors agree to be accountable for all aspects of the work in ensuring that questions related to the accuracy or integrity of any part of the work are appropriately investigated and resolved. JTO'B and IDG are guarantors of the study.

Funding GE Healthcare provided funding and administrative support for this pooled analysis. All coauthors (except IDG and PFS, who were GE employees at the time the paper was prepared) contributed independent of the sponsor, as noted above, and retained full editorial control of the content and the decision to publish.

Competing interests JTO'B reports grants and other from GE Healthcare, grants and other from Lilly, other from Bayer Healthcare, other from TauRx, other from Cytox, outside the submitted work. WHO reports grants and personal fees from GE Healthcare, personal fees from Amersham.Buchler, outside the submitted work. IGM reports grants and personal fees from GE Healthcare, outside the submitted work. DGG reports grants and personal fees from GE Healthcare, during the conduct of the study. ZW reports personal fees from GE Healthcare, personal fees from Bayer Healthcare, grants from GE Healthcare, grants from Lundbeck, other from GE Healthcare and personal fees from Novartis, outside the submitted work. KT reports grants and personal fees from GE Healthcare, outside the submitted work. ET reports grants from The Michael J Fox Foundation for Parkinson's Research, personal fees from Novartis, TEVA, Boehringer Ingelheim, UCB, Solvay, Lundbeck, TEVA, outside the submitted work. PFS reports other (salary) from GE Healthcare, during the conduct of the study; other (salary) from GE Healthcare, outside the submitted work. IDG reports employment from GE Healthcare, during the conduct of the study.

Ethics approval Ethics Committees or Institutional Review Boards approved the protocol and amendments for each study (see online supplementary table S2).

Provenance and peer review Not commissioned; externally peer reviewed.

Data sharing statement No additional data are available.

Open Access This is an Open Access article distributed in accordance with the Creative Commons Attribution Non Commercial (CC BY-NC 3.0) license, which permits others to distribute, remix, adapt, build upon this work noncommercially, and license their derivative works on different terms, provided the original work is properly cited and the use is non-commercial. See: http:// creativecommons.org/licenses/by-nc/3.0/

\section{REFERENCES}

1. Litvan I, Bhatia KP, Burn DJ, et al. Movement Disorders Society Scientific Issues Committee report: SIC Task Force appraisal of clinical diagnostic criteria for Parkinsonian disorders. Mov Disord 2003:18:467-86.

2. McKeith IG, Galasko D, Kosaka K, et al. Consensus guidelines for the clinical and pathologic diagnosis of dementia with Lewy bodies (DLB): report of the consortium on DLB international workshop. Neurology 1996;47:1113-24.

3. Hyman BT, Phelps $\mathrm{CH}$, Beach TG, et al. National Institute on Aging-Alzheimer's Association guidelines for the neuropathologic assessment of Alzheimer's disease. Alzheimers Demen 2012;8:1-13.

4. Sorbi S, Hort J, Erkinjuntti T, et al. EFNS-ENS Guidelines on the diagnosis and management of disorders associated with dementia Eur J Neurol 2012;19:1159-79.

5. Filippi M, Agosta F, Barkhof F, et al. EFNS task force: the use of neuroimaging in the diagnosis of dementia. Eur J Neurol 2012;19: e131-501.

6. Bajaj N, Hauser RA, Grachev ID. Clinical utility of dopamine transporter single photon emission CT (DaT-SPECT) with (123l) ioflupane in diagnosis of parkinsonian syndromes. $J$ Neurol Neurosurg Psychiatry 2013;84:1288-95.

7. Litvan I, Maclntyre A, Goetz CG, et al. Accuracy of the clinical diagnoses of Lewy body disease, Parkinson disease, and dementia with Lewy bodies: a clinicopathologic study. Arch Neurol 1998;55:969-78.

8. Tatsch K, Poepperl G. Nigrostriatal dopamine terminal imaging with dopamine transporter SPECT: an update. J Nucl Med 2013;54:1331-8.

9. McKeith IG, Ballard CG, Perry RH, et al. Prospective validation of consensus criteria for the diagnosis of dementia with Lewy bodies. Neurology 2000;54:1050-8.

10. Lopez OL, Becker JT, Kaufer DI, et al. Research evaluation and prospective diagnosis of dementia with Lewy bodies. Arch Neurol 2002;59:43-6.

11. European Medicines Agency prescribing information for DaTSCAN Internet 2013. http://www.ema.europa.eu/docs/en GB/document library/EPAR_-_Product_Information/human/000266/WC500035355. pdf (accessed 21 Aug 2013).

12. Full Prescribing Information for DaTscan (US). Internet 2013. http:// www3.gehealthcare.com/en/Products/Categories/Nuclear_Imaging Agents/ /media/Downloads/us/Product/Product-Categories/ Nuclear-Imaging-Agents/DaTscan/GEHealthcare_DaTscanPrescribing-Information.pdf (accessed 21 Aug 2103).

13. Benamer HTS, Patterson J, Grosset DG, et al. Accurate differentiation of parkinsonism and essential tremor using visual assessment of [1231]-FP-CIT SPECT imaging: the [1231]-FP-CIT study group. Mov Disord 2000;15:503-10.

14. McKeith I, O'Brien J, Walker Z, et al. Sensitivity and specificity of dopamine transporter imaging with 123I-FP-CIT SPECT in dementia with Lewy bodies: a phase III, multicentre study. Lancet Neurol 2007;6:305-13. 
15. O'Brien JT, McKeith IG, Walker Z, et al. Diagnostic accuracy of 123I-FP-CIT SPECT in possible dementia with Lewy bodies. $\mathrm{Br} J$ Psychiatry 2009;194:34-9.

16. Marshall VL, Reininger CB, Marquardt M, et al. Parkinson's disease is overdiagnosed clinically at baseline in diagnostically uncertain cases: a 3-year European multicenter study with repeat [123I] FP-CIT SPECT. Mov Disord 2009;24:500-8.

17. Catafau AM, Tolosa E. Impact of dopamine transporter SPECT using 123I-ioflupane on diagnosis and management of patients with clinically uncertain Parkinsonian syndromes. Mov Disord 2004;19:1175-82.

18. Tolosa E, Borght TV, Moreno E. Accuracy of DaTSCAN (123l-ioflupane) SPECT in diagnosis of patients with clinically uncertain parkinsonism: 2-year follow-up of an open-label study. Mov Disord 2007;22:2346-51.

19. Walker Z, Jaros E, Walker RW, et al. Dementia with Lewy bodies: a comparison of clinical diagnosis, FP-CIT single photon emission computed tomography imaging and autopsy. J Neurol Neurosurg Psychiatry 2007;78:1176-81.

20. Antonini A, Benti R, De NR, et al. 123l-loflupane/SPECT binding to striatal dopamine transporter (DAT) uptake in patients with Parkinson's disease, multiple system atrophy, and progressive supranuclear palsy. Neurol Sci 2003;24:149-50.

21. Bairactaris C, Demakopoulos N, Tripsianis G, et al. Impact of dopamine transporter single photon emission computed tomography imaging using I-123 ioflupane on diagnoses of patients with parkinsonian syndromes. J Clin Neurosci 2009;16:246-52.

22. Colloby SJ, Firbank MJ, Pakrasi S, et al. A comparison of $99 \mathrm{mTC}$-exametazime and 123I-FP-CIT SPECT imaging in the differential diagnosis of Alzheimer's disease and dementia with Lewy bodies. Int Psychogeriatr 2008;20:1124-40.

23. Doepp F, Plotkin M, Siegel L, et al. Brain parenchyma sonography and 123I-FP-CIT SPECT in Parkinson's disease and essential tremor. Mov Disord 2008;23:405-10.

24. Eshuis SA, Jager PL, Maguire RP, et al. Direct comparison of FP-CIT SPECT and F-DOPA PET in patients with Parkinson's disease and healthy controls. Eur J Nucl Med Mol Imaging 2009;36:454-62.

25. Garcia Vicente AM, Vaamonde CJ, Poblete Garcia VM, et al. [Utility of dopamine transporter imaging (123-I ioflupane SPECT) in the assessment of movement disorders]. Rev Esp Med Nucl 2004:23:245-52.

26. Goethals I, Ham H, Dobbeleir A, et al. The potential value of a pictorial atlas for aid in the visual diagnosis of 123I FP-CIT SPECT scans. Nuklearmedizin 2009;48:173-8.

27. Kahraman D, Eggers C, Holstein A, et al. 123I-FP-CIT SPECT imaging of the dopaminergic state. Visual assessment of dopaminergic degeneration patterns reflects quantitative $2 \mathrm{D}$ operator-dependent and 3D operator-independent techniques. Nuklearmedizin 2012;51:244-51.

28. Koch W, Hamann C, Radau PE, et al. Does combined imaging of the pre- and postsynaptic dopaminergic system increase the diagnostic accuracy in the differential diagnosis of parkinsonism? Eur J Nucl Med Mol Imaging 2007;34:1265-73.

29. Lorenzo BC, Miquel RF, Roca BI, et al. [Differential diagnosis of parkinsonism using dopamine transporters brain SPECT]. Med Clin (Barc) 2004;122:325-8.

30. Martinez del Valle Torres MD, Ramos ME, Amrani RT, et al. [Functional assessment of nigro-striatal pathway with FP-CIT in patients with multiple system atrophy subtype C]. Med Clin (Barc) 2011;137:440-3

31. Morgan S, Kemp P, Booij J, et al. Differentiation of frontotemporal dementia from dementia with Lewy bodies using FP-CIT SPECT. J Neurol Neurosurg Psychiatry 2012;83:1063-70.

32. O'Brien JT, Colloby S, Fenwick J, et al. Dopamine transporter loss visualized with FP-CIT SPECT in the differential diagnosis of dementia with Lewy bodies. Arch Neurol 2004;61:919-25.

33. Ortega Lozano SJ, Martinez del Valle Torres MD, Jimenez-Hoyuela Garcia JM, et al. [Diagnostic accuracy of FP-CIT SPECT in patients with parkinsonism]. Rev Esp Med Nucl 2007;26:277-85.

34. Ortega Lozano SJ, Martinez del Valle Torres MD, Jimenez-Hoyuela Garcia JM, et al. [Diagnostic accuracy of FP-CIT SPECT in the evaluation of patients with clinically uncertain parkinsonian syndrome]. Neurologia 2007;22:86-92.

35. Papathanasiou N, Rondogianni P, Chroni P, et al. Interobserver variability, and visual and quantitative parameters of (123)I-FP-CIT SPECT (DaTSCAN) studies. Ann Nucl Med 2012;26:234-40.

36. Pifarre P, Cuberas G, Hernandez J, et al. Cortical and subcortical patterns of $\mathrm{I}-123$ iodobenzamide SPECT in striatal $\mathrm{D}(2)$ receptor parkinsonisms. Clin Nucl Med 2010;35:228-33.
37. Piperkova E, Georgiev R, Daskalov M, et al. The brain scintiscan with iodine-123-ioflupane to diagnose early Parkinson's disease; seven months follow up. First results in Bulgaria. Hell $\mathrm{J} \mathrm{Nucl} \mathrm{Med}$ 2006:9:31-5.

38. Suarez-Pinera M, Prat ML, Mestre-Fusco A, et al. [Interobserver agreement in the visual and semi-quantitative analysis of the 123I-FP-CIT SPECT images in the diagnosis of Parkinsonian syndrome]. Rev Esp Med Nucl 2011;30:229-35.

39. Sudmeyer M, Antke C, Zizek T, et al. Diagnostic accuracy of combined FP-CIT, IBZM, and MIBG scintigraphy in the differential diagnosis of degenerative parkinsonism: a multidimensional statistical approach. J Nucl Med 2011:52:733-40.

40. Van LK, Casteels C, De CL, et al. Dual-tracer dopamine transporter and perfusion SPECT in differential diagnosis of parkinsonism using template-based discriminant analysis. J Nucl Med 2006;47:384-92

41. Van LK, De CL, Dom R, et al. Dopamine transporter SPECT using fast kinetic ligands: 123I-FP-beta-CIT versus 99mTc-TRODAT-1. Eur J Nucl Med Mol Imaging 2004;31:1119-27.

42. Vlaar AM, de Nijs T, Kessels AG, et al. Diagnostic value of 123 l-ioflupane and 123I-iodobenzamide SPECT scans in 248 patients with parkinsonian syndromes. Eur Neurol 2008;59:258-66.

43. Hauser RA, Bajaj N, Marek K, et al. Sensitivity, specificity, positive and negative predictive values and diagnostic accuracy of DaTscan ${ }^{\mathrm{TM}}$ (ioflupane 1123 injection): predicting clinical diagnosis in early clinically uncertain parkinsonian syndrome. J Neurol Stroke 2014;1:00003

44. Papathanasiou ND, Boutsiadis A, Dickson J, et al. Diagnostic accuracy of (1)(2)(3)I-FP-CIT (DaTSCAN) in dementia with Lewy bodies: a meta-analysis of published studies. Parkinsonism Relat Disord 2012;18:225-9.

45. Vlaar AM, van Kroonenburgh MJ, Kessels AG, et al. Meta-analysis of the literature on diagnostic accuracy of SPECT in parkinsonian syndromes. BMC Neurol 2007;7:27.

46. Gorovets A, Marzella L, Rieves D, et al. Efficacy considerations for U.S. Food and Drug Administration approval of diagnostic radiopharmaceuticals. J Nucl Med 2013;54:1479-84.

47. McKeith IG, O'Brien JT, Ballard C. Diagnosing dementia with Lewy bodies. Lancet 1999;354:1227-8.

48. Kis B, Schrag A, Ben-Shlomo Y, et al. Novel three-stage ascertainment method: prevalence of $P D$ and parkinsonism in South Tyrol, Italy. Neurology 2002;58:1820-5.

49. Schrag A, Ben-Shlomo Y, Quinn N. How valid is the clinical diagnosis of Parkinson's disease in the community? J Neurol Neurosurg Psychiatry 2002;73:529-34.

50. Newman EJ, Breen K, Patterson J, et al. Accuracy of Parkinson's disease diagnosis in 610 general practice patients in the West of Scotland. Mov Disord 2009;24:2379-85.

51. Kupsch AR, Bajaj N, Weiland F, et al. Impact of DaTscan SPECT imaging on clinical management, diagnosis, confidence of diagnosis, quality of life, health resource use and safety in patients with clinically uncertain parkinsonian syndromes: a prospective 1-year follow-up of an open-label controlled study. J Neurol Neurosurg Psychiatry 2012;83:620-8.

52. Hughes AJ, Ben-Shlomo Y, Daniel SE, et al. What features improve the accuracy of clinical diagnosis in Parkinson's disease: a clinicopathologic study. 1992. Neurology 2001;57:S34-8.

53. Hughes AJ, Daniel SE, Lees AJ. Improved accuracy of clinical diagnosis of Lewy body Parkinson's disease. Neurology 2001:57:1497-9.

54. Berardelli A, Wenning GK, Antonini A, et al. EFNS/MDS-ES/ENS recommendations for the diagnosis of Parkinson's disease. Eur $J$ Neurol 2013;20:16-34.

55. Djang DS, Janssen MJ, Bohnen N, et al. SNM practice guideline for dopamine transporter imaging with 123I-ioflupane SPECT 1.0 J Nucl Med 2012:53:154-63.

56. NICE Clinical Guideline 35: Parkinson's disease diagnosis and management in primary and secondary care, June 2006. Internet 2006. http://www.nice.org.uk/nicemedia/live/10984/30088/30088.pdf (accessed 21 Aug 2013)

57. Diagnosis and pharmacological management of Parkinson's disease: a national clinical guideline. Internet 2010. http://www.sign. ac.uk/guidelines/fulltext/113/index.html (accessed 21 Aug 2013).

58. Parkinson Progression Marker Initiative. The Parkinson Progression Marker Initiative (PPMI). Prog Neurobiol 2011;95:629-35.

59. Kalra S, Grosset DG, Benamer HT. Differentiating vascular parkinsonism from idiopathic Parkinson's disease: a systematic review. Mov Disord 2010;25:149-56.

60. Oh M, Kim JS, Kim JY, et al. Subregional patterns of preferential striatal dopamine transporter loss differ in Parkinson disease, 
progressive supranuclear palsy, and multiple-system atrophy. J Nucl Med 2012;53:399-406.

61. Colloby SJ, McParland S, O'Brien JT, et al. Neuropathological correlates of dopaminergic imaging in Alzheimer's disease and Lewy body dementias. Brain 2012;135:2798-808.

62. Iranzo A, Valldeoriola F, Lomena F, et al. Serial dopamine transporter imaging of nigrostriatal function in patients with idiopathic rapid-eye-movement sleep behaviour disorder: a prospective study. Lancet Neurol 2011;10:797-805.

63. Stiasny-Kolster K, Doerr Y, Moller JC, et al. Combination of 'idiopathic' REM sleep behaviour disorder and olfactory dysfunction as possible indicator for alpha-synucleinopathy demonstrated by dopamine transporter FP-CIT-SPECT. Brain 2005;128:

126-37. 